\title{
Vertebral Osteomyelitis: Unexpected Cause of Back Pain
}

\author{
Fermin Lopez-Rivera ${ }^{\mathrm{a}, \mathrm{b}}$, Xavier Colon Rivera ${ }^{\mathrm{a}}$, \\ Hernan Gonzalez Monroig ${ }^{\mathrm{a}}$
}

\begin{abstract}
Osteomyelitis is an inflammation of bone caused by a pyogenic organism. Osteomyelitis is one of the most important musculoskeletal infections, commonly occurring in long bones. Vertebral osteomyelitis is fairly rare and is often overlooked. It is usually acquired hematogenously, although there are other ways of infection. Only about half of patients develop fever $>38^{\circ} \mathrm{C}$ and symptoms are unspecific. The most common organism is Staphylococcus aureus, with Pseudomonas aeruginosa being an unexpected pathogen accounting for about $6 \%$. We present a case of a 56-year-old Hispanic male that visited emergency room presenting with back pain of 3 weeks of evolution being discharged home. Patient returned 2 days after with no improvement. Blood test returned under normal range. Physical exam showed a focal tenderness at thoracic level. Blood culture recovered Pseudomonas aeruginosa sensitive to levofloxacin. CT/MRI was performed and showed vertebral osteomyelitis. Antibiotic was started for 6 weeks. Therapy was guided with erythrocyte sedimentation rate (ESR)/C-reactive protein (CRP). Patient was evaluated monthly for 6 months and improved. Vertebral osteomyelitis is a challenging diagnosis. The consequences of the disease are dire if not diagnosed on time. Historically, the diagnosis of vertebral osteomyelitis remains in a positive culture of bone biopsy. Nowadays, when handling a suspected hematogenous vertebral osteomyelitis, blood culture has gained importance. Whether a blood culture recovered a pathogen, therapy should be based on sensitivity and can be guided following ESR/CRP levels. This type of management can preclude invasive and expensive methods, such as bone biopsy.
\end{abstract}

Keywords: Bacteremia; Discitis; Osteomyelitis

\section{Introduction}

Osteomyelitis is an inflammation of bone caused by a pyogenic organism. Osteomyelitis is one of the most important musculoskeletal infections. The signs and symptoms of vertebral osteo-

Manuscript submitted July 3, 2017, accepted July 18, 2017

anternal Medicine Department; San Juan City Hospital, San Juan, Puerto Rico ${ }^{b}$ Corresponding Author: Fermin Lopez-Rivera, Internal Medicine Department, San Juan City Hospital, 550 Calle Jazmin Cotto Laurel, San Juan, Puerto Rico. Email: drlopezrivera.ga@gmail.com

doi: https://doi.org/10.14740/jmc2870w myelitis are non-specific. Only about half of patients develop fever $>38^{\circ} \mathrm{C}$. However, some symptoms are non-specific and include weakness, malaise, backache and non-specific pain. Back pain is the second most common symptom-related reason for clinician visits in the United States [1]. Up to $85 \%$ of adults have low back pain at some point in their lives $[1,2]$. Osteomyelitis is commonly occurring in long bones, thus vertebral osteomyelitis is a challenging diagnosis, fairly rare and is often overlooked. It is an infection of the disk space and the adjacent vertebral bodies clinically characterized by back pain, fever, and, sometime neurological disorders [3]. The age distribution of this disease is bimodal: one peak at age less than 20 years and a second peak in the subjects aged 50 - 70 years. The most common microorganism implicated in the pyogenic vertebral osteomyelitis is Staphylococcus aureus and Pseudomonas aeruginosa responsible for 6\% [4]. Risk factors for P. aeruginosa include recent hospitalization, recent broad-spectrum antibiotic use, coexisting pulmonary disease, immunocompromised and intravenous drug abuse (IVDA). Most cases of vertebral osteomyelitis result from hematogenous seeding from a distant source such as the urinary tract, skin, infected vascular catheters, endocarditis, or bursitis/septic arthritis [5].

\section{Case Report}

A 56-year-old Hispanic male that was on his usual state of health that consisted of independence on activities of daily living until 3 weeks ago visited the emergency room with a 3 -week history of backache being discharged home. Patient returned 2 days after to our institution with no improvement. Blood test taken at emergency room showed white blood cells of $8.9 \times 10^{9} / \mathrm{L}$, hemoglobin of $12.3 \mathrm{~g} / \mathrm{dL}$, and platelets of 348 $\times 10^{9} / \mathrm{L}$. Electrolytes showed no derangement, amylase of 37 and lipase of 53, returning under normal range. Renal function, calculated with Cockcroft, was $>83 \mathrm{~mL} / \mathrm{min}$. Vital signs were temperature of $37.1{ }^{\circ} \mathrm{C}$, blood pressure of $108 / 76$, heart rate of 78 , and respiratory rate of 17 . The patient stated the backache was oppressive and band-like across the middle back, 6/10 in intensity, constant, non-radiating, worsened with movement, and was not relieved with ibuprofen. The past medical history was remarkable for chronic HCV and former IVDA. At physical exam patient was normotensive, eupneic and afebrile. Tenderness to palpation was noted at T5-T8 level. Neurologic exam showed no deficits, and strength was $5 / 5$ in all extremities. Patient was admitted with diagnosis of suspected vertebral osteomyelitis, based on history and physical exam. Once admitted, further laboratories showed an elevated erythrocyte 


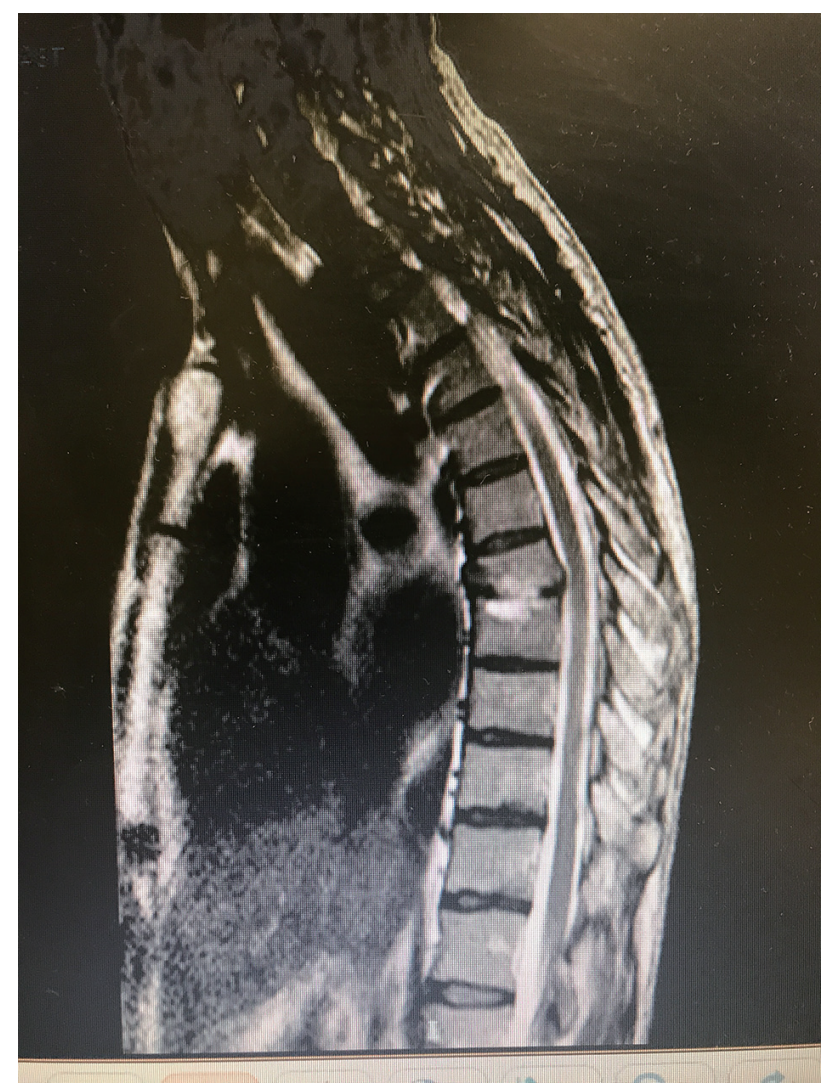

Figure 1. MRI of the thoracic spine demonstrated abnormal signal intensity of the T7-T8 vertebral bodies and T7-T8 intervertebral disc, representing diskitis osteomyelitis with associated compression deformity and mild spinal canal stenosis.

sedimentation rate $(\mathrm{ESR}, 57 \mathrm{~mm} / \mathrm{h})$ with no elevation of white blood cells and no shifting. C-reactive protein (CRP) returned at $16.5 \mathrm{mg} / \mathrm{dL}$. HIV was negative. Blood cultures showed growth of $P$. aeruginosa in two bottles. Electrocardiogram showed normal sinus rhythm with no significant abnormalities and the QTc was 406 ms. Posterior-anterior chest X-ray and lateral views showed clear lung field and osseous abnormality. Abdomen and pelvic CT without contrast showed no abnormality. Furtherly patient underwent a thoracic CT scan that showed endplate lucencies marginating the T7-T8 disc level and anterior wedging consistent with discitis/osteomyelitis. Later, an MRI of the thoracic spine showed abnormal signal intensity of the T7-T8 vertebral bodies and T7-T8 intervertebral disc, representing diskitis osteomyelitis with associated compression deformity and mild spinal canal stenosis (Fig. 1). Upon admission, broad-spectrum IV antibiotics were started (vancomycin and meropenem) before blood cultures. Infectious disease was consulted recommending deescalate therapy to levofloxacin $750 \mathrm{mg}$ IV daily for 42 days. ESR and CRP decreased with antibiotic regimen. Subsequent blood cultures showed no bacterial growth. No surgical procedure was performed given the lack of neurological signs. Patient was evaluated monthly for 6 months with physical exam and ESR/CRP. Aforementioned markers were repeated every month as well and returned under normal range.

\section{Discussion}

Osteomyelitis is inflammation of the bone caused by an infecting organism. Vertebral osteomyelitis is a challenging diagnosis, often delayed several months and may initially be misdiagnosed when non-specific findings predominate the history [6]. The incidence is estimated to be approximately 2.4 cases per 100,000 overall [7]. There is a clear age-dependent increase from 0.3 cases/100,000 at ages less than 20 years to 6.5 cases $/ 100,000$ at ages more than 70 years [7]. The following diseases can increase the incidence: diabetes mellitus, malnourishment, substance abuse, cancer, immunocompromised patient, chronic kidney disease, and chronic liver disease. Osteomyelitis can be classified with the Waldvogel classification system that divides osteomyelitis into the categories of hematogenous, contiguous and chronic [8]. There is a second system known as the Cierny-Mader classification, based on anatomic, clinical, and radiologic features [9].

Although all bones are subject to infection, the lower extremity is most commonly involved. Vertebral osteomyelitis is commonly monomicrobial, with $S$. aureus being the most common organism isolated $[5,10]$. S. aureus causes $40-50 \%$, strep $12 \%$ and $20 \%$ by gram-negative bacilli (9\%) [7]. P. aeruginosa causes $6 \%$ of osteomyelitis. The signs and symptoms of vertebral osteomyelitis are non-specific. Only about half of patients develop fever $>38{ }^{\circ} \mathrm{C}$. However, some symptoms include weakness, malaise and non-specific pain. We state, in regard to our patient, it was caused by nerve compression at T7-T8 level, which improved after treatment. The diagnosis was achieved due to high suspicion based on physical exam. Plain radiographs initially showed soft tissue changes, muscle swelling, and blurring of the soft tissue planes. The first change in bone indicates that the infection has been present for at least 3 weeks. Osteomyelitis must extend at least $1 \mathrm{~cm}$ and compromise $30-50 \%$ of bone mineral content to produce noticeable changes in plain radiographs [11]. Cross-sectional imaging modalities such as CT scanning and MRI are now considered standard in the diagnosis of osteomyelitis. Although expensive, they are sensitive and specific. These modalities give excellent anatomic delineation of the infected area and the surrounding soft tissues. The MRI remains greater sensitivity, specificity and accuracy for the detection of osteomyelitis [12]. ESR and CRP are highly sensitive, and help to guide therapy [13]. Osteomyelitis treatment is evolving. Blood cultures have become a cornerstone and are very useful because they can preclude invasive procedures, if the pathogen is recovered [14]. During the classic bone biopsy, the needle may break the bone or injure a nerve, blood vessel or organ nearby. Antimicrobial regimen must be directed against the pathogen recovered in the blood culture. Vertebral osteomyelitis caused by gram-negative organism, such as $P$. aeruginosa, responds to old quinolones (ciprofloxacin and levofloxacin). Newer agents are not recommended in the treatment of infections caused by $P$. aeruginosa [15]. Trials showed osteomyelitis is predominantly caused by Enterobacteriaceae or P. aeruginosa, and clinical improvement rates ranged from $65 \%$ to $100 \%$ [16]. The optimal duration of therapy is unclear; most authors recommend therapy is between 4 and 6 weeks [17]. 


\section{Conclusion}

Vertebral osteomyelitis must remain as a high differential diagnosis in every patient with a localized musculoskeletal pain. It is a defiant diagnosis, bearing in mind the infrequency of disease. Diagnosis is based on physical findings, laboratories, and imaging studies. Despite of all modern technologies, physical exam must remain the primary tool to suspect osteomyelitis. Years ago, the diagnosis of vertebral osteomyelitis remains in a positive culture of bone biopsy. Nowadays, when handling a suspected hematogenous vertebral osteomyelitis, blood culture has gained importance. Whether a blood culture recovers a pathogen, therapy should be based on sensitivity and can be guided following CRP levels. We hypothesize this type of management can preclude invasive methods (for diagnosis and follow-up), avoiding morbidities.

\section{Acknowledgments}

The authors wish to acknowledge the assistance of LopezMieres, MD and Fernando Abreu, MD attendings the Internal Medicine program of San Juan City Hospital, in the diagnosis, guidance of management and the preparation of the manuscript of this article.

\section{Financial Support}

No source of financial support to disclose.

\section{Conflicts of Interest}

We have no conflicts of interest to declare.

\section{References}

1. Deyo RA, Tsui-Wu YJ. Descriptive epidemiology of low-back pain and its related medical care in the United States. Spine (Phila Pa 1976). 1987;12(3):264-268.

2. Cassidy JD, Carroll LJ, Cote P. The Saskatchewan health and back pain survey. The prevalence of low back pain and related disability in Saskatchewan adults. Spine (Phila Pa 1976). 1998;23(17):1860-1866; discussion 1867.

3. Zimmerli W. Clinical practice. Vertebral osteomyelitis. N Engl J Med. 2010;362(11):1022-1029.
4. Butler JS, Shelly MJ, Timlin M, Powderly WG, O'Byrne JM. Nontuberculous pyogenic spinal infection in adults: a 12-year experience from a tertiary referral center. Spine (Phila Pa 1976). 2006;31(23):2695-2700.

5. McHenry MC, Easley KA, Locker GA. Vertebral osteomyelitis: long-term outcome for 253 patients from 7 Cleveland-area hospitals. Clin Infect Dis. 2002;34(10):13421350.

6. Abram SR, Tedeschi AA, Partain CL, Blumenkopf B. Differential diagnosis of severe back pain using MRI. South Med J. 1988;81(12):1487-1492.

7. Longo DL, Fauci AS, Kasper DL, Hauser SL, Jameson J, Loscalzo J. eds. Osteomyelitis. Harrison's Principles of Internal Medicine, 18e. New York, NY: McGraw-Hill; 2012. p. 158.

8. Cierny G 3rd, Mader JT, Penninck JJ. A clinical staging system for adult osteomyelitis. Clin Orthop Relat Res. 2003;414:7-24.

9. Cierny G, Mader JT, Pennick JJ. A clinical staging system for adult osteomyelitis. Contemp Orthop. 1985;10:17-37.

10. Lew DP, Waldvogel FA. Osteomyelitis. N Engl J Med. 1997;336(14):999-1007.

11. Pineda C, Espinosa R, Pena A. Radiographic imaging in osteomyelitis: the role of plain radiography, computed tomography, ultrasonography, magnetic resonance imaging, and scintigraphy. Semin Plast Surg. 2009;23(2):8089.

12. Meyers SP, Wiener SN. Diagnosis of hematogenous pyogenic vertebral osteomyelitis by magnetic resonance imaging. Arch Intern Med. 1991;151(4):683-687.

13. Markanday A. Acute phase reactants in infections: evidence-based review and a guide for clinicians. Open Forum Infect Dis. 2015;2(3):ofv098.

14. Calhoun JH, Manring MM, Shirtliff M. Osteomyelitis of the long bones. Semin Plast Surg. 2009;23(2):59-72.

15. Jones RN, Sader HS, Beach ML. Contemporary in vitro spectrum of activity summary for antimicrobial agents tested against 18569 strains non-fermentative Gram-negative bacilli isolated in the SENTRY Antimicrobial Surveillance Program (1997-2001). Int J Antimicrob Agents. 2003;22(6):551-556.

16. Howard W, Biedenbach DJ, Jones RN. Comparative antimicrobial spectrum and activity of the desfluoroquinolone BMS284756 (T-3811) tested against non-fermentative Gram-negative bacilli. Clin Microbiol Infect. 2002;8(6):340-344.

17. Conterno LO, da Silva Filho CR. Antibiotics for treating chronic osteomyelitis in adults. Cochrane Database Syst Rev. 2009;3:CD004439. 